\title{
Application of standard micro-anatomical staining methods to epoxy resin-embedded sections
}

\author{
S. R. APARICIO AND P. MARSDEN \\ From the Department of Pathology, School of Medicine, Leeds
}

SYNOPSIS Staining of semi-thin sections of osmium-fixed epoxy resin (Epon or Araldite)-embedded tissues with haematoxylin and acid dyes as counterstains (eosin or phloxin) was successfully achieved after intense oxidation using hydrogen peroxide as the oxygen donor. Results are closely similar to those obtained with paraffin sections (haematoxylin and eosin): nuclei stain blue, cytoplasm and extracellular structures ( $e g$, collagen, elastica, basement membranes) stain with varying shades of red. Preliminary studies show that similarly good results, comparable with standard paraffinembedded histological materials, can be obtained, using $\mathrm{H}_{2} \mathrm{O}_{2}$ pretreatment, with Weigert's iron haematoxylin, Mallory's phosphotungstic acid haematoxylin, Alcian blue, PAS reaction, Sudan III, Sudan black, oil red, Gomori's aldehyde fuchsin, and Baker's acid haematein.

Staining of semi-thin sections of osmium-fixed, epoxy resin-embedded tissues was developed in the last decade, mainly using basic aniline dyes in neutral or alkaline solutions (Bencosme, Stone, Latta, and Madden, 1959; Moore, Mumaw, and Schoenberg, 1960; Richardson, Jarett, and Finke, 1960; Trump, Smuckler, and Benditt, 1961 ; Grimley, Albrecht, and Michelitch, 1965; Lee and Hopper, 1965; Aoki and Gutierrez, 1967; Aparicio and Marsden, 1969; Huber, Parker, and Odland, 1968). Counterstaining with ordinary haematoxylin and acid dyes has proved to be almost useless, and although debatable, this is probably due to the presence of osmium in the tissues. Munger (1961) succeeded in staining with Ehrlich's haematoxylin, after preliminary treatment of methacrylate sections with peracetic acid. However, when the method was applied to Epon sections he found that the duration of staining had to be increased from two to five times longer than for methacrylate. Apparently Munger's method has not become a routine method for semi-thin sections, probably because of the lengthy procedure involved. Schwalbach, Lickfeld, and Hoffmeister (1963) reported success in staining Vestopal with some of the iron haematoxylins. However, Vestopal appears to be much easier to stain than Epon or Araldite. Apart from these two reports no further work on the haematoxylin staining techniques appears to have been reported

Received for publication 31 January 1969. since Epon and Araldite became universally adopted as embedding media.

This communication reports a simple and efficient method of obtaining haematoxylin-eosin and haematoxylin-phloxin epoxy resin preparations identical in colour contrast with paraffin sections.

\section{MATERIAL AND METHODS}

A variety of tissues (human brain, peripheral nerve, kidney, liver, tongue, striated muscle, and lymph node; guinea-pig brain, muscle, and liver; rat aorta and mouse ovary) were fixed for electron microscopy in glutaraldehyde, ranging from 2.5 to $6.5 \%$ concentration in phosphate buffer (Sabatini, Bensch, and Barrnett, 1963) or in $10 \%$ buffered formaldehyde, post-osmicated in $2 \%$ osmium tetroxide buffered with phosphate. After suitable dehydration, blocks were then embedded in Epon (Luft, 1961) or Araldite (Glauert and Glauert, 1958). Semi-thin sections were obtained at 0.5 to $1 \mu$ with a Porter-Blum MT-1 ultramicrotome and were attached to clean glass slides by heating on a hot plate as described previously (Aparicio and Marsden, 1969).

STAINING Our basic modification of the method lies in a rapid and intense oxidation of the sections using hydrogen peroxide as the oxygen donor. For haematoxylin-eosin and haematoxylin-phloxin staining proceed as follows:-

1 Immerse slides in a Coplin jar containing fresh $15 \% \mathrm{H}_{2} \mathrm{O}_{2}$ for 10 minutes.

2 Wash thoroughly in tap water.

3 Stain in Harris's haematoxylin (Lillie, 1965) for 15 minutes. 
4 Wash in tap water and blue in tap water alkalinized with a few drops of ammonium hydroxide.

5 Wash in tap water.

6 Counterstain in $1 \%$ aqueous eosin Y ( 2 drops of concentrated acetic acid added per Coplin jar) for five minutes. Alternatively, counterstain with $1 \%$ aqueous phloxin (G. T. Gurr) for two minutes.

7 Wash, dry, and mount in DPX.

If the sections are not firmly and thoroughly attached to the glass slides, the oxygen bubbles released will lift them and the precipitation of collected stain will ruin the preparation. Although thicker sections (yellow interference colour or above) yield better intensity of staining they are more prone to this type of precipitation hazard. However, spreading in $10 \%$ acetone and adequate heating ensure even attachment to the slide. Experience shows that the staining time for haematoxylin may have to be increased with human postmortem material. Counterstaining with eosin may sometimes be difficult owing to the affinity of Epon with eosin and because of increased basophilia of the cytoplasm after oxidation. However, acidification of the eosin solution overcomes these problems easily. Phloxin can be extremely useful as a counterstain and indeed in certain cases, $e g$, brain, yields better results than eosin. Erythrosin at $1 \%$ concentration can also be used. Araldite is more difficult to stain than Epon and staining has to be increased from twice to three times.

The following staining methods were also used after pretreatment with $\mathrm{H}_{2} \mathrm{O}_{2}$ : Weigert's iron haematoxylin, Alcian blue, PAS reaction, Sudan III, Sudan black, oil red, Gomori's aldehyde fuchsin, Mallory's phosphotungstic acid haematoxylin, Bodian copper protargol, and Baker's acid haematein. These techniques were used according to the procedures described in Lillie's Histopathologic Technic and Practical Histochemistry (1965), with no special modification other than adjusting the staining times for maximum colour contrast.

\section{RESULTS}

With haematoxylin-eosin nuclei stain deep blue as in paraffin sections. Cytoplasm stains with varying shades of red according to the degree of cytoplasmic acidophilia of different cells. Figures 1 to 3 illustrate only a few examples: striated muscle fibres (Fig. 1), myelin sheaths, oligodendrocytes, luteal cells (Fig. 2), macrophages (Fig. 3), hepatocytes and erythrocytes stain red. With these thin $(0.5 \mu)$ sections interesting differential intensities of eosin staining, eg, different cytoplasmic granules, which are masked in the much thicker $(2$ to $7 \mu$ ) ordinary histological sections, are consistently seen. Saturated lipids, as in the ovary, remain unstained and the lipid vacuoles can be seen against the red cytoplasmic matrix (Fig. 2). Extracellular collagen, elastin, basement membranes, and reticulin fibres take varying shades of red.

As far as the other methods used were concerned, failure was only met with the Bodian silver method. The deposition of silver in the axons was so small as

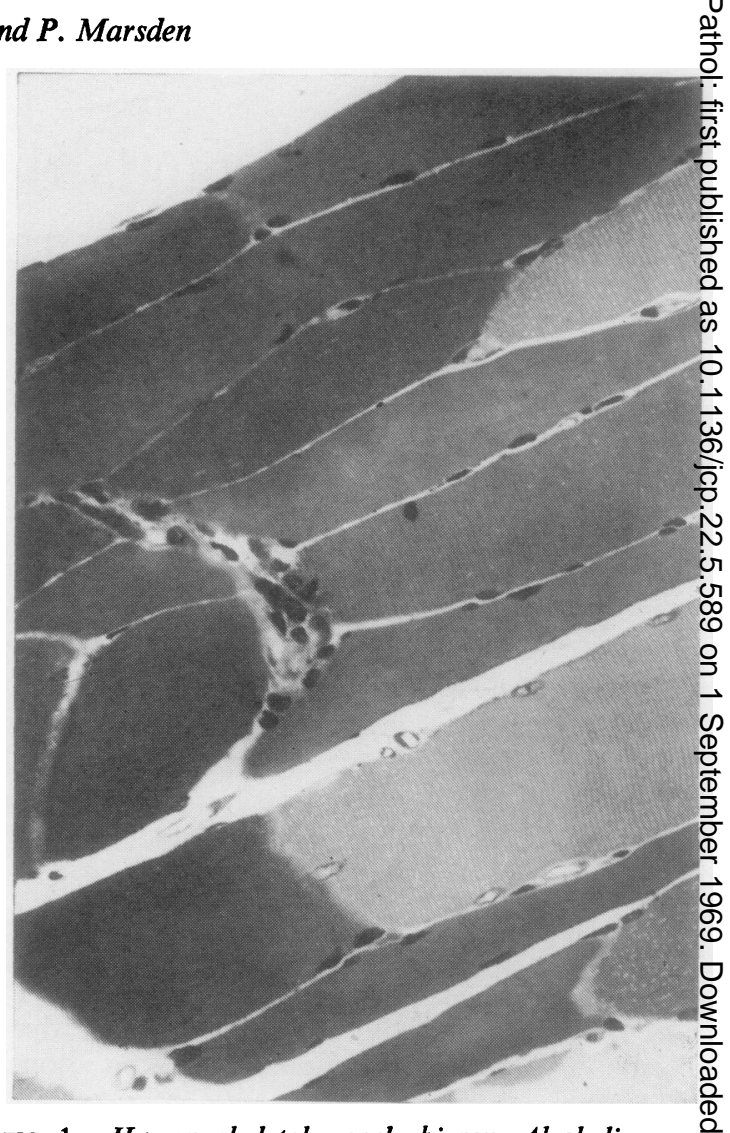

FIG. 1. Human skeletal muscle biopsy. Alcoholic myo pathy. Healthy fibres show more intense cytoplasmi $\overline{\bar{\theta}}$ acidophilia (the darker fibres) and deeper nuclear baso philia in contrast with paler fibres showing early sign? of cytoplasmic and nuclear degeneration. Glutaraldehyde? osmium. Epon. Haematoxylin-eosin $\times 400$.

to be of no real practical value. However, a slightly more intense deposit was found in hypertrophic. glial fibres. The Baker's acid haematein test gave its specific reaction with myelin sheaths but the colour contrast with this type of preparation was reduced because of impregnation of the embedding resin with the acid haematein. The PAS reaction. (Fig. 4) yielded brightly coloured reaction products much superior to those obtained in preparations without previous oxidation with $\mathrm{H}_{2} \mathrm{O}_{2}$. The more danted haematoxylin techniques gave excellentw nuclear and cytoplasmic resolution and were easily carried out. Weigert's iron haematoxylin was ese pecially useful as a counterstain for the PAS reaction (Fig. 4). Amongst the lipid stains the best results were obtained with Sudan black, especially whenए applied to sections of white matter of the centraP nervous system. The oil red stain when applied to sections of the ovary stained some of the luteal cel $\underline{B}$ lipids with a reddish brown colour. 


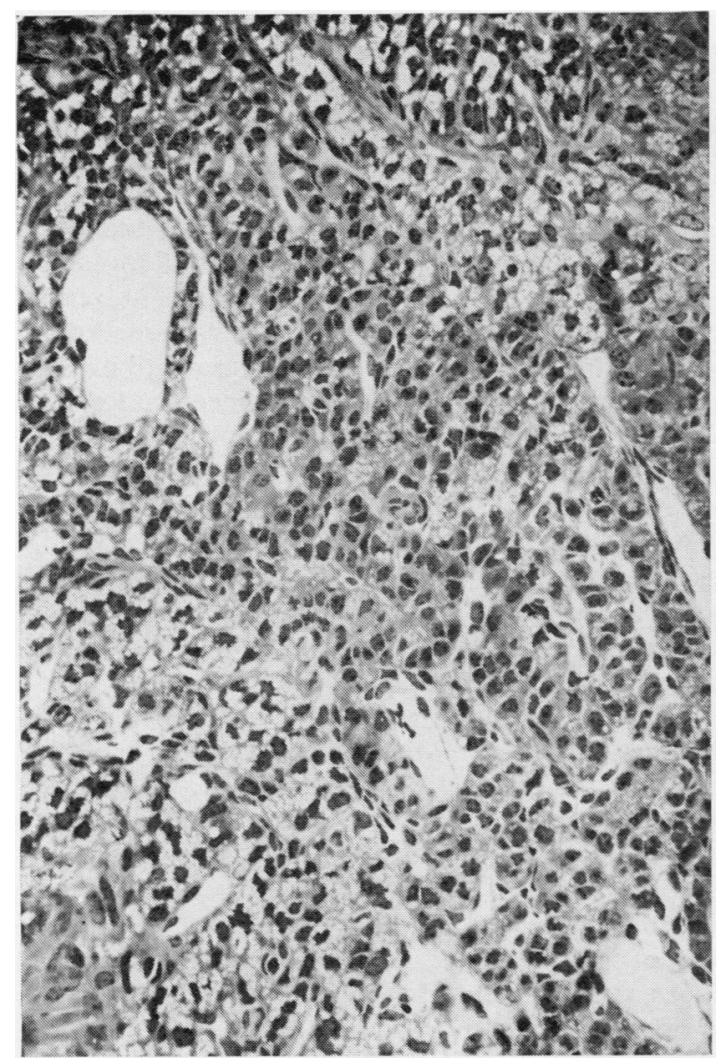

FIG. 2.

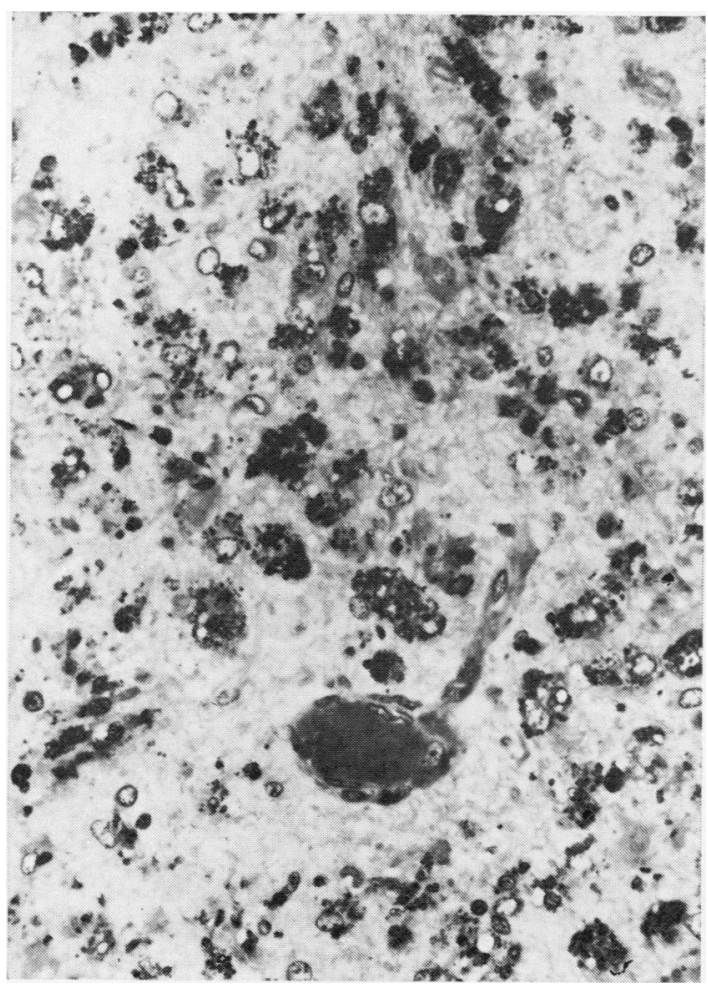

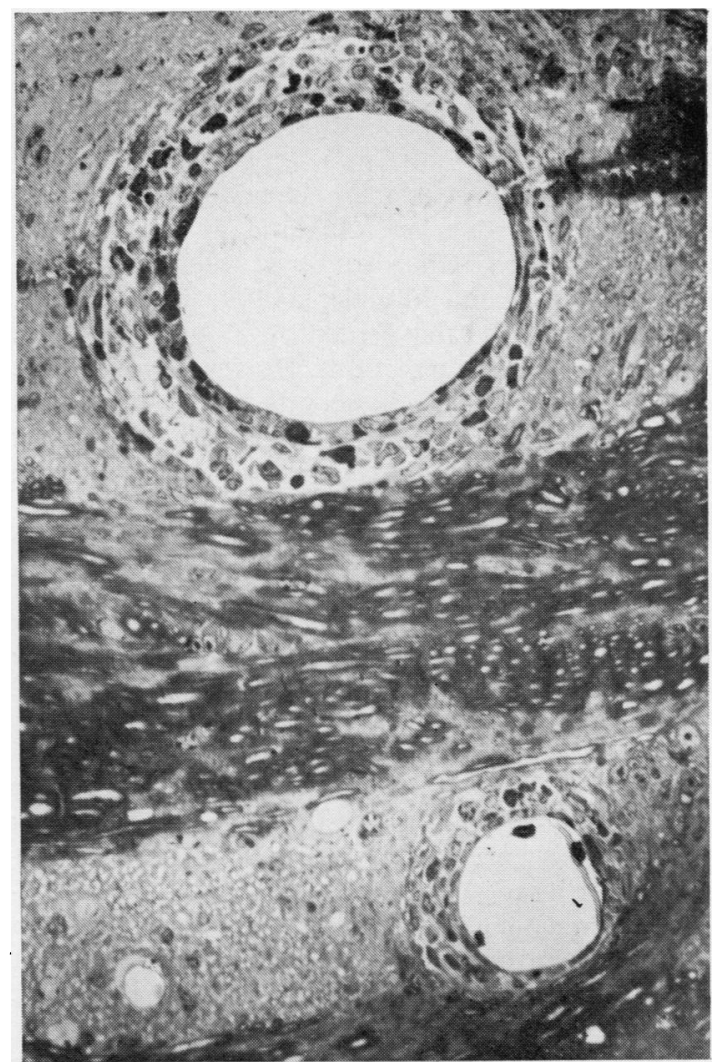

FIG. 3.

FIG. 2. Mouse ovary. Normal. Corpus luteum in regression. Solid, alveolus-like groups of luteal cells are seen invested by sinusoidal blood capillaries, ie, an endocrine cell pattern is still retained here. Nuclei with typical chromatin clumps are stained blue and cytoplasm stains pale red. Lipid vacuoles (saturated lipids) are unstained. Erythrocytes are bright red. Glutaraldehyde-osmium. Epon. Haematoxylin-eosin $\times 400$.

FIG. 3. Guinea-pig periventricular white matter in experimental allergic encephalomyelitis. Two perivenular cuffs of inflammatory cells are seen. Note intense cytoplasmic acidophilia in some of the macrophages. Perithelial cells show paler blue nuclei and pale pink cytoplasm. Myelin stains bright red. Glutaraldehyde perfusionosmium. Epon. Haematoxylin-phloxin $\times 400$.

FIG. 4. Edge of plaque of multiple sclerosis in cortical white matter (necropsy material). An active perivenular lesion, showing gitter cells laden with PAS-positive (here, deep black), myelin-derived lipids. Note the paler hypertrophied astrocytes. Glutaraldehyde-osmium. Epon. $P A S$ Weigert's iron haematoxylin $\times 400$. 


\section{DISCUSSION}

The haematoxylin-eosin or haematoxylin-phloxin method described is not as rapid as some of the monochromatic (Richardson et al, 1960) or dichromatic (Aparicio and Marsden, 1969; Huber et al, 1968) methods that have been developed as routine staining for semi-thin sections. However, none of the basic aniline dye methods yield the histologically and cellular colour contrasts achieved with the present procedure; nor do they offer direct correlation between semi-thin and paraffin sections.

Obviously the successful application of the routine histological staining methods to osmium-fixed epoxy-resin-embedded tissues lies partially in the release of the osmium blockade to reactive groups. Like Munger (1961) we found that intense oxidation can be used successfully for this purpose. However, on the evidence presented by Munger, it would appear that peracetic acid is only mildly effective for Epon-embedded tissues. He found that staining times had to be extended up to four times longer than those used for methacrylate, even after prolonged peracetic acid treatment. Our results indicate that hydrogen peroxide is much more efficient in a much shorter time, and the staining times thereafter are shorter.

Since the osmium reaction during fixation of the tissues is not yet fully understood the method is used empirically. It is assumed that part of the bound osmium will be reoxidized and enters into solution as osmium tetroxide but perhaps the formation of chelated compounds cannot be excluded entirely. We are at the moment conducting tests designed to clarify this point. Surprisingly, the intense oxidation applied to the sections does not substantially inactivate the chemical groups binding the stains. Such inactivation will occur, however, if the oxidation is carried too far, as our preliminary experiments on the optimum pretreatment time suggested. Obviously one aims at achieving a compromise between removal of osmium and inactivation. As our experience shows, this should be kept in mind, especially when performing histochemical reactions such as the PAS reaction. In the latter instance the pretreatment should be kept as short as possible to prevent further oxidation of the dialdehydes responsible for the reaction.

The preliminary results obtained with staining methods other than haematoxylin-eosin seem to흘 indicate that the $\mathrm{H}_{2} \mathrm{O}_{2}$ oxidation of osmium-fixed, resin-embedded tissues may be used as a universal $\mathbb{\Phi}_{\square}^{\mathbb{\Phi}}$ pretreatment before these various staining methods. N The usefulness of this pretreatment seems to extend. beyond the application of general microanatomical ${ }^{\circ}$ methods into more specific histochemical methods, $\overrightarrow{\vec{\omega}}$ $e g$, PAS reaction, Baker's acid haematein and alciano blue for mucopolysaccharides. However, methods? such as the silver methods, which are heavilyin dependent on the amount of stainable biological material present in any given section, are probablyir bound to failure owing to the extremely low thick-o ness of the resin sections.

The results obtained with some of the lipid stainsobviously call for a more detailed investigation to bes dealt with elsewhere. It is, however, interesting? to note that the oil red method as used for frozen? sections can be applied successfully to epoxy resin sections after $\mathrm{H}_{2} \mathrm{O}_{2}$ oxidation. It seems probable that ${ }^{-}$ the lipids, the extraction of which was prevented $\overrightarrow{0}$ by the osmium fixation during processing of thee blocks, were rendered available (after $\mathrm{H}_{2} \mathrm{O}_{2}$ oxidation)O for oil red staining.

\section{REFERENCES}

Aoki, A., and Gutierrez, L. S. (1967). Stain Technol., 42, 307. Aparicio, S. R., and Marsden, P. (1969). J. Micros., 89, 139.

Bencosme, S. A., Stone, R. S., Latta, H., and Madden, S. C. (1959) J. biophys. biochem. Cytol., $5,508$.

Glauert, A. M., and Glauert, R. H. (1958). Ibid, 4, 191.

Grimley, P. M., Albrecht, J. M., and Michelitch, H. J. (1965). Stain Technol., 40, 357.

Huber, J. D., Parker, F., and Odland, G. F. (1968). Ibid, 43, 83.

Lee, J. C., and Hopper, J., Jr. (1965). Ibid, 40, 37.

Lillie, R D. (1965). Histopathologic Technic and Practical Histochemistry. 3rd ed. McGraw-Hill Book Company.

Luft, J. H. (1961). J. biophys. biochem. Cytol., 9, 409.

Moore, R. D., Mumaw, V., and Schoenberg, M. D. (1960). J. Ultrastruct. Res., 4, 113

Munger, B. L. (1961). J. biophys. biochem. Cytol., 11, 502.

Richardson, K. C., Jarett, L., and Finke, E. H. (1960). Stain Technol. 을

Sabatini, D. D., Bensch, K., and Barrnett, R. J. (1963). J. cell. Biol.

Schwalbach, G., Lickfeld, K. G., and Hoffmeister, H. (1963). Stain Technol., 38, 15.

Trump, B. F., Smuckler, E. A., and Benditt, E. P. (1961). J. Ultra struct. Res., 5, 343. 